Onomástica Desde América Latina, n.4, v.2, julho - dezembro, 2021, p. 4 -31. ISSN 2675-2719

https://doi.org/10.48075/odal.v0i0.27485

\title{
Naming System of Kèdainiai Women in Local Register Books of the 17th-18th Centuries
}

\section{Sistema nomeador das mulheres de Kèdainiai nos livros de registros locais dos séculos XVII e XVIII}

\author{
Alma Ragauskaitè \\ Institute of the Lithuanian Language \\ https://orcid.org/0000-0001-5952-8027 \\ alma.ragauskaite@lki.lt
}

\begin{abstract}
Municipal register books, inventories, and church registers are among the key historical anthroponymic sources of Lithuanian townspeople. The most notable among these sources are 24 register books of Kèdainiai written in the Latin and Polish languages. The data of research are 148 recordings of Kedainiai women. The article aims to establish and compare the naming system (of women) used in Kedainiai of the 17th-18th centuries, underlining more characteristic Polonisation events of their personal names. The Polonisation of Lithuanian anthroponyms are among the main factors affecting their records. The following major research methods are used to discuss the data of historical anthroponymy: descriptive, reconstructive and comparative. In the 17 th century, women were mostly recorded using four anthroponyms, and in the 18th century, only two personal names (i.e., a first name and a surname or replacing the latter with an anthroponym) were used. Traditional Christian names were predominant among the studied recordings.
\end{abstract}

Keywords: women of Kèdainiai; register books; historical anthroponymy; Lithuanian historical anthroponym; naming; word-formation.

Resumo: Livros de registros municipais, inventários e registros de igreja fazem parte das fontes históricas antroponímicas mais importantes de citadinos lituanos. Entre essas fontes, a mais notável é o conjunto de 24 livros de registro de Kèdainiai escritos na língua latina e na língua polonesa, no qual há 148 registros de nomes de mulheres desta localidade. O objetivo deste artigo é identificar e comparar os sistemas de nomeação de mulheres utilizados no município nos séculos XVII e XVIII, enfatizando-se os fenômenos mais característicos da polonização de seus nomes pessoais, haja vista ser um dos principais fatores que influenciaram o registro desses nomes. Nesta pesquisa para a discussão dos dados antroponímico históricos, foram adotados, majoritariamente, os métodos de pesquisa descritivo, reconstrutivo e comparativo. Os resultados mostram que, nos registros estudados, enquanto, no século XVII, a maioria das mulheres era registrada com quatro antropônimos, no século XVIII, elas eram registradas com apenas dois nomes (um prenome e um sobrenome ou um antropônimo usado no lugar do sobrenome), os dados também mostram que nomes cristãos tradicionais predominaram.

Palavras-chave: mulheres de Kèdainiai; livros de registros; antroponímica histórica; antropônimos históricos lituanos; nomeação; formação de palavras. 
Onomástica Desde América Latina, n.4, v.2, julho - dezembro, 2021, p. 4 -31. ISSN 2675-2719

https://doi.org/10.48075/odal.v0i0.27485

\section{Introduction}

In the Grand Duchy of Lithuania ${ }^{1}$ of the 17 th-18th centuries, Kedainiai, with its protected and fostered multicultural traditions, was among the few private towns owned by the aristocratic family of the Radziwills. In the 17th century, Kedainiai was a multi-religious and multinational town, an important centre of the Evangelical Reformats and the written Lithuanian language. Investigations have already commenced into the old multinational onomastics of Kedainiai. First, the discussion focused on trends of the seventeenth and eighteenth centuries, particular to the recording of Scottish nationals. The greater variety was particular to the personal names of Scottish women of Kèdainiai. Their Latinised and Slavicised names could be modified differently in various inscriptions of record files of the magistrate, consequently resulting in a significant quantity of graphical variations of surnames. The personal names of Scottish women of Kèdainiai had Slavic suffixes -ova and -ovna. ${ }^{2}$ In about 1628-1629, people of Scottish origin had arrived at Kèdainiai already possessing surnames; however, the recording of their hereditary anthroponyms in register books of the town varied. This situation can be explained by the absence of a uniform mandatory naming system and the writing tradition of the time. Nevertheless, the conclusion was made that the recordings of names of the seventeenth and eighteenth centuries clearly attest to the functioning of Scottish hereditary anthroponyms of Kèdainiai residents in local historical documents (RAGAUSKAITE், 2016a: 56-57).

\footnotetext{
${ }^{1}$ A description of Lithuanian anthroponymic system can be found in English in: Ragauskaite (2008; 2021); Seide \& Petrulione (2018); in Spanish in Seide \& Petrulionè (2020) and in Portuguese in Amaral \& Seide (2020).

${ }^{2} 1652$ pani Czapmanowa mieszczanka kiejdańska; 1659 pani Forbeżowa; 1679 Halszka Hallibortonowna; 1679 P(ani) Gertruda Kiuckowa; 1679 P(ani) Katarzina Krechtonowna; 1688 Janowa Heiowa a teraznieyfza Jerzyna Mollefonowa; 1659 panni Margareta Simsonowna; 1750 Domina Anna Forsejowa vidua; 1736 Pani Elzbieta z Barklaiow Halibortonowa; 1731 Katarzyna z Arnetow primi voti Eliziufzowa Reiowa ad prcesens Rofzowa; 1717 Anna Mozeniufzowa Jerzyna Sumerwiłowa.
} 
Onomástica Desde América Latina, n.4, v.2, julho - dezembro, 2021, p. 4 -31. ISSN 2675-2719 https://doi.org/10.48075/odal.v0i0.27485

It is believed that in 1629, Kristupas Radvila invited Germans (artisans, merchants etc.) to Kedainiai. The inscription of their names lacked uniformity. This phenomenon was affected by the specificity of investigated register books and the context of inscriptions. The personal names of German women of Kedainiai also had Slavic suffixes -ova and -ovna ${ }^{3}$ (RAGAUSKAITE், 2016b: 117-120). The analysis of trends specific to female names of Kèdainiai Germans determined that registers of christenings, marriages and deaths of the town's Lutheran church recorded female German names rather authentically. Besides, female German names were recorded without the Slavic suffixes -ova, -ovna, which is different from Kedainiai administrative sources of the seventeenth and eighteenth centuries ${ }^{4}$ (RAGAUSKAITÉ, 2018a: 180). As evidenced, several ethnic groups ${ }^{5}$ of the community that resided in Kedainiai had different attributes of the naming system, surname origin and structure, hence confirming the relevance and the need to continue further investigations into the historical anthroponymy of Kèdainiai residents of the seventeenth and eighteenth centuries.

This article aims to establish and compare the naming trends of local Kèdainiai community members (mostly Lithuanian and Polish women) in the 17th-18th centuries, underlining more characteristic phenomena and the most important factors particular to the

\footnotetext{
${ }^{3} 1782$ Honesta Eufrosina primi voti Ryttbergerowa; 1786 Anna Marianna z Lemmow Gusmanowa Wdowa Obyw(atelka) Miasta Kieydan; 1795 Rozalia Guttszteynowa; 1752 Stawetna Katarzyna Margarita Mejerowa Maystrowa Kunsztu Tokarskiego; 1743 Pani Plecowa; 1767 Rydbergerowa; 1795 Panni Anna Szulcowna.

41840 Katharina Bigang; 1840 Karolinę Bodendorf; 1840 Johanna Feltz; 1870 Emilie Fucks; 1840 Maria Golz; 1840 Friderica Grau; 1840 Elisabeth Grimm; 1853 Adeline Heckendorf; 1840 Caroline Helwich; 1858 Anna Herrmann; 1866 Annette Keller; 1840 Wilhelminę Lackner; 1862 Bertha Laune; 1868 Adolphina Leinart; 1842 Anna Susanna Ludwig; 1848 Catharine Luther; 1840 Katharinę Meynę; 1840 Katharina Riegel; 1869 Dorothea Schlüter; 1840 Amalię Schoenborm; 1851 Louise Schönborn; 1840 Christina Straus; 1840 Dorothea Weimar; 1873 Emma Zoch.

${ }^{5}$ For more information about the ethnic and religious composition of the population of Kedainiai in the seventeenth and eighteenth centuries, see e.g. ŠINKŪNAS, 1928: 21-36; TWOREK, 1966: 193-194; 1969: 213; ŽIRGULIS, 2002a: 130-134; RAGAUSKAITE், 2016a: 47-49; 2016b: 113-115; 2017: 54-56; 2018a: 175-176.
} 
Onomástica Desde América Latina, n.4, v.2, julho - dezembro, 2021, p. 4 -31. ISSN 2675-2719 https://doi.org/10.48075/odal.v0i0.27485

Polonisation of Lithuanian personal names, which impacted the recording of Kèdainiai residents in local administrative sources.

The anthroponymic material for the article was mostly collected from historical town documents of the seventeenth and eighteenth centuries. ${ }^{6}$ With a major part of Kedainiai Magistrate books of the seventeenth century lost in a fire, the early inscriptions are greatly missed (ŽIRGULIS, 2002b: 24). The Office of the Chief Archivist of Lithuania is in possession of 24 register books of Kèdainiai written in Latin and Polish languages. These books are among the most significant sources of historical anthroponymy of Kèdainiai residents (TYLA, 2002: 27; RAGAUSKAITE், 2008: 29). A major part of the register books involves inscriptions reflecting various town events and matters of the local community (inheritance, mortgage, sales and purchase agreements, debt documents, guild statutes etc.). Protocol books of magistrate sessions, meetings and town courts date back to 1759 , in several sets of 1759-1763, 1767-1773, 17771792 (TYLA, 2000: 65). The volume of books varies. The largest register book dates back to 1756-1758 and contains 627 pages, while the smallest has only 84 pages and contains inscriptions of 1732-1740. Clerks responsible for town register books had sets of formulas they partly followed to record different people and court-case decisions (JABLONSKIS, 1979: 222). It was calculated that the documents were written by seventeen clerks (TYLA, 2000: 73-75), e.g. 1665 Woyciech Kalinowski Pisarz Kieydanski KAK 51v $^{7}$; 1744 Melcher Bowk Pisarz Magdeburgij Kieydanskiey $\mathrm{KAK}_{7}$ 2v; 1769 P(an $)^{8}$ Adam Ciołkiewicz Pisarz $\mathrm{KAK}_{15} 78 ; 1724$

\footnotetext{
${ }^{6}$ See the list of archival sources at the end of the article.

${ }^{7}$ All anthroponyms presented in the article are registered. Every name has the indication of the year of recording, the abbreviation of the historical source and the page number. The presentation of anthroponyms of Kedainiai residents aims to preserve the original writing, which is different to the tradition of historical source publication (based on LEPSZY, 1953: 7-15; WOLFF, 1957: 155-181).

${ }^{8}$ Abbreviations recorded in historical documents were supplemented. The insertion indicated in brackets.
} 
Onomástica Desde América Latina, n.4, v.2, julho - dezembro, 2021, p. 4 -31. ISSN 2675-2719 https://doi.org/10.48075/odal.v0i0.27485

Symona Czyzewskiego Pisarza Magdeburyi Kieydan(skiei) gen. sg. ${ }^{9} \mathrm{KAK}_{4}$ 107; 1759 Pan Jozeff Dumaszewicz ktory podziękowawszy Miastu za Elekcya Mieysce Pisarskie KAK 10 1; 1762 Marcinem Grybassem Pisarzem abl. sg. KAK $923 ; 1770$ Szlachetny <..> P(an) Jan Gulbin Pisarz $\mathrm{KAK}_{15}$ 90; 1763 Stanistawem Lubenem Protunc Pisarzem y Calym Szlachetnym Magistratem abl. sg. KAK 14 23; 1771 Pan Ignacy Lukaszewicz Pisarz KAK 16 7; 1765 Mikołajem Judabanem Markowskim Pisarzem y Catym Szlachetnym Magistratem abl. sg. KAK 14 221; 1704 Pana Adriana Miloszewskiego Pisarza <... Kieydanskiego gen. sg. KAK 3 4v; 1762 Stefanem Mixiewiczem Protunc Pisarzem abl. sg. $\mathrm{KAK}_{13} 41 ; 1794$ Benedykt Rudelewicz Pisarz Magistratu Miasta $<\ldots>$ wolnego Kieydan $\mathrm{KAK}_{24}$ 142; 1756 Jozefem Antonim z Bończa Rudkowskim przysieglym Magdeburyi Kieydanskiey Pisarzem abl. sg. KAK 12105 - 1753 Jozefem Antonim z Boncza Rutkowskim Pisarzem abl. sg. KAK 11 109; 1774 Michat Symonowicz Protunc Pisarz KAK $_{10}$ 16; 1764 Kazimierzem Walentynowiczem Protunc Pisarzem abl. sg. KAK 14107.

The following major research methods are used to discuss the data of historical anthroponymy: descriptive, comparative and reconstructive (RAGAUSKAITÉ, 2005: 31; 2021: 2). The choice of these methods is determined by the objectives of the article. It will be necessary to describe in detail the collected data and to compare the naming trends of Kedainiai women of the 17th and 18th centuries. The article will also present the reconstructed women's anthroponyms with Lithuanian suffixes. The recordings of women were selected to demonstrate functioning anthroponyms with affixes of Lithuanian formation (Lithuanian stems or endings and suffixes) or Slavic formants. From the discussed historical town documents of the 17th-18th centuries, recordings of 148 Kèdainiai women were selected. Different types of naming were

\footnotetext{
${ }^{9}$ See the list of another abbreviations: abl. - Lat. (cāsus) ablätīvus "ablative case"; comp. - compare; curt. current; dat. - Lat. (cāsus) datīvus "dative case"; gen. - Lat. (cāsus) genetīvus "genitive case"; Lat. - Latin; n. name; Pol. - Polish; sg. - Lat. (numerus) singulāris "singular"; sur. - surname.
} 
Onomástica Desde América Latina, n.4, v.2, julho - dezembro, 2021, p. 4 -31. ISSN 2675-2719 https://doi.org/10.48075/odal.v0i0.27485

calculated. Surnames and personal names used in their stead were the primary focus of this investigation.

\section{Recording of Women in the 17 th century}

It is important to accurately determine the anthroponyms attributable to female residents of Kedainiai, which are present in the historical documents of the 17th century. The sources do not provide their naming in the form of separate lists but rather in a varied context of recording inscriptions (RAGAUSKAITĖ, 2000: 94; 2003: 82; 2004: 8-9; 2006: 81-82; 2014: 9). Female local members of the Kèdainiai community are marked as mieszczanka, obywatelka in Polish, e.g., 1690 Ja iagnie/zka Kazimierzowa Baltromieiewiczowa <...> Mie/zczanka Miasta Kieydan $\mathrm{KAK}_{2} 20 ; 1689$ Zofia Jasułayciowna Andrzeiowa Jutewiczowa MieJzczanka Miasta Kieydan KAK $_{1}$ 81; 1691 Ja Anna Jerzyna Marcinkiewiczowna Pronowska Obywatelka Miafta Kieydan $\mathrm{KAK}_{2} 46$.

The manuscripts were written in Polish and Latin. Hence, the anthroponyms of Lithuanian women were Slavicized and could be modified in different records of the documents. Certain facts of Slavicization of personal names were distinguished in the historical documents under analysis: sound substitution, use of Slavic suffixes in the anthroponyms of Lithuanian origin, omission of endings, etc. (MACIEJAUSKIENĖ, 1994: 25-30; RAGAUSKAITĖ, 1999: 148-149; 2005: 61-66; 2015: 63-64; 2018b: 57-58; 2019: 182-183). Based on these principles, personal names of Kèdainiai residents were analysed or reconstructed. The restored forms of anthroponyms were compared with current Lithuanian surnames.

Thirty-three different naming instances of Kedainiai women (using two, three, four or five personal names) were taken from Kedainiai historical sources of the 17th century. The first 
Onomástica Desde América Latina, n.4, v.2, julho - dezembro, 2021, p. 4 -31. ISSN 2675-2719 https://doi.org/10.48075/odal.v0i0.27485

members of these naming instances were Christian names. Most frequently used were traditional Polish or Latin forms of Christian names. In total, 13 different names were counted. Graphic variants of these historical names were also compared to current Lithuanian names, e.g., 1633 Anna $\mathrm{KAK}_{1} 5^{10}$. Comp. curt. n. Onà (LVKŽ 294) ${ }^{11}$; 1691 Barbara KAK 2 33. Comp. curt. n. Barborà (LVKŽ 90); 1668 Elzbieta $\mathrm{KAK}_{1}$ 67. Comp. curt. n. Elzbietà (LVKŽ 148); 1690 Ewa $\mathrm{KAK}_{2} 24 \mathrm{v}$. Comp. curt. n. Ievà (LVKŽ 195); 1690 Helena $\mathrm{KAK}_{2}$ 31. Comp. curt. n. Elenà (LVKŽ 146); 1690 iagnie/zka $\mathrm{KAK}_{2}$ 20. Comp. curt. n. Agnètè (LVKŽ 62); 1690 Iudycie dat. sg. $\mathrm{KAK}_{2}$ 13v. Comp. curt. n. Judità (LVKŽ 212); 1691 Jadwiga $\mathrm{KAK}_{2}$ 34. Comp. curt. n. Jadvygà, Jadvýga (LVKŽ 201); 1690 Katharzyna KAK 2 7v. Comp. curt. n. Kotrynà (LVKŽ 235); 1690 Konftantia $\mathrm{KAK}_{2}$ 13v. Comp. curt. n. Konstáncija (LVKŽ 234); 1690 Luciey dat. sg. KAK 2 13v. Comp. curt. n. Liùcija, Lùcija (LVKŽ 252); 1690 Marianna $\mathrm{KAK}_{2}$ 10. Comp. curt. n. Marijonà

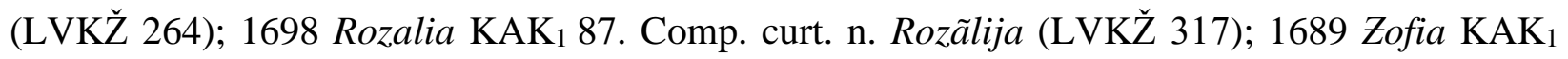
81; 1690 Zofia $\mathrm{KAK}_{2}$ 13; 1691 Zophia $\mathrm{KAK}_{2}$ 34v. Comp. curt. n. Zòfija (LVKŽ 385).

Personal names of suffixal formation after the husband or father followed the first name. Anthroponyms after the father were formed using the suffix -ova (-owa). A surprisingly small number of recordings represented women who were unmarried, young adults or teenagers. They were rarely named in these documents. The instances usually related to the death of parents, in the case of the orphanhood of underaged children or relatives. Personal names after the father were mostly formed using the suffix -ovna (-owna). Townswomen with names containing Polish

\footnotetext{
${ }^{10}$ Names registered in their form according to the alphabet. Different name forms are indicated, denoting only one page alongside, on which this form was mentioned. An exhaustive register of name occurrence would greatly expand the volume of this article.

${ }^{11}$ All current names provided in the article are registered (LVKŽ - Kuzavinis, K. \& Savukynas, B. (1994). Lietuviu vardu kilmés žodynas. Vilnius: Mokslo ir enciklopedijų leidykla).
} 
Onomástica Desde América Latina, n.4, v.2, julho - dezembro, 2021, p. 4 -31. ISSN 2675-2719 https://doi.org/10.48075/odal.v0i0.27485

suffixes of the -sk- type were named after the husband or father (RAGAUSKAITE், 2005: 107; 2015: 63).

In the 17th century, Kedainiai townswomen were most frequently recorded using four anthroponyms (Christian name, personal name after the father and two anthroponyms after the husband). In total, 15 such instances were found (45.46\% of all naming instances), e.g.: 1691 Uczciwey Paniey Katarzynie Abramowskiey Macieiowey Lapienayciowey dat. sg. $\mathrm{KAK}_{2} 26$; 1694 Katarzynie Jasułayciownie Macieiowey Lawrynowiczowey dat. sg. $\mathrm{KAK}_{1}$ 85v; 1691 Zophia Jerominowna Woyciechowa Mątwiłowiczowa $\mathrm{KAK}_{2}$ 47v; $1690<\ldots>$ Ja Ewa Butkiewiczowna Janowa Pawlowiczowa $\mathrm{KAK}_{2}$ 21; 1691 Ja Anna Jerzyna Marcinkiewiczowna Pronowska Obywatelka Miafta Kieydan(skiego) $\mathrm{KAK}_{2}$ 46; 1698 <...> Rozalia Wiszniewska Stephanowa Rakiewiczowa $\mathrm{KAK}_{1}$ 87; 1691 Zophia Kurklinska Stanisławowa Staginska KAK 2 26. 1690 Katarzyna Dawgielowna Jerzyna Trabfzewiczowa $\mathrm{KAK}_{2} 15 \mathrm{v}$.

Apart from this most productive naming model, Kèdainiai townswomen were recorded using other ways as well. Seven women $(21.21 \%)$ were named using two personal names, e.g.: 1690 Ja Ewa Jankowska KAK224v; 1691 Ja Zophia Kunkulewiczowa KAK 2 34; 1690 Spectabilis D(omi)na Helifabetha Kuturowiczowna $\mathrm{KAK}_{2}$ 4v; 1690 Iudycie Męzynfkiey dat. sg. $\mathrm{KAK}_{2} 13 \mathrm{v} ; 1688$ Katarzyna Miksiewiczowna $\mathrm{KAK}_{1}$ 78; 1691 Jadwiga Tarandziowna $\mathrm{KAK}_{2} 34$.

Seven naming instances $(21.21 \%)$ were found having three anthroponyms, i.e., a first name and two personal names after the husband or father, e.g.: 1690 Iagnieszka Kazimierzowa Baltromieiewiczowa <...> Mie/3czanka Miasta Kieydan $\mathrm{KAK}_{2}$ 20; 1690 Ewa Krzy/ztofowa Dziadowiczowa $\mathrm{KAK}_{2}$ 29; 1691 Paniey Zofiey Woyciechowey Mątwilowiczowey dat. sg. $\mathrm{KAK}_{2}$ 46v; 1634 Elzbiecie Woyciechowney Ptaciakowney dat. sg. KAK $17 ; 1633$ Ja Anna 
Onomástica Desde América Latina, n.4, v.2, julho - dezembro, 2021, p. 4 -31. ISSN 2675-2719 https://doi.org/10.48075/odal.v0i0.27485

Grzegorzewna Ruczkayciowna <...> Mieszczanka Miasta <...> Kieydan $\mathrm{KAK}_{1}$ 5; 1690 Helena Jurewiczowna Wolucka $\mathrm{KAK}_{2} 31$.

Four $(12.12 \%)$ Kèdainiai townswomen were recorded using five personal names, e.g.: 1691 Barbara Adamowna Baiorowiczowa Bartłomieiowa Ju/sewiczowa $\mathrm{KAK}_{2}$ 33; 1690 Konftantia Perfka Mikolaiowa Kazimierzowa Męźynfka KAK $213 \mathrm{v} ; 1690$ Katharzyna Michniewiczowna Adrzeiowna Janowa Wysocka Miesczanka Miasta Kieidan $\mathrm{KAK}_{2} 7 \mathrm{v}$.

As evidenced, Slavic suffixes -ova and -ovna were predominant among the different naming types of Keddainiai townspeople of the 17th century. However, among the inscriptions of the 17th century, some personal names of townspeople also had the Lithuanian suffix -aičia (written as -aycia) (RAGAUSKAITE், 2014: 15), e.g.: *Eivaičia: 1690 Ja Anna Jeywaycia Macieiowa Garnelowa Mieszcz(an)ka Miafta Kieydan $\mathrm{KAK}_{2}$ 16. Comp. curt. sur. Éiva, Eivà (LPŽ I 570) ${ }^{12}$; *Ja s a ič i a : $1690<$ <..> Ja Zofia Jafaycia $\mathrm{KAK}_{2}$ 4. Comp. curt. sur. Jãsas (LPŽ I 813); *Plauškaičia: $1690<\ldots>$ Pani Zofia Plawfzkaycia Piotrowa Mordafzewiczowa $\mathrm{KAK}_{2}$ 13. Comp. curt. sur. Plauškà (LPŽ II 469). Anthroponyms of unmarried women containing the suffix -yčia could have been formed from the male personal names with endings $-i s$, $-y s$; and those with the suffix -aičia - from male anthroponyms with endings - $a$, -as (MACIEJAUSKIENE, 1991: 268-271). Nevertheless, the historical sources were varied and inconsistent in this respect (RAGAUSKAITE், 2015: 65).

The given examples of surnames Polonisation demonstrated that authentic Lithuanian anthroponyms of Kèdainiai women were modified in different ways. Therefore, in the spoken language, these women were referred to differently than recorded in the analysed sources.

\footnotetext{
${ }^{12}$ All current surnames provided in the article are registered (LPŽ I, II - Maciejauskienè, V.; Razmukaite, M. \& Vanagas, A. (Ed.) (1985-1989). Lietuviu pavardžiu žodynas (Vol. 1-2). Vilnius: Mokslas.
} 
Onomástica Desde América Latina, n.4, v.2, julho - dezembro, 2021, p. 4 -31. ISSN 2675-2719 https://doi.org/10.48075/odal.v0i0.27485

Clearly, this attests to interlaced traditions of the Polish written and Lithuanian spoken languages. The article presented the most characteristic distinctive features particular to the recording of personal names of Kèdainiai women based on inscriptions in historical documents rather than the live language. This was affected by the particularity of analysed historical sources, their context, the competence of clerks, and the different social status of registered individuals. Long protocols of meetings and court sessions were handwritten, possessing very few recording formulas. In these large-scale historical manuscripts, names and surnames were only elements of the text.

\section{Recording of Women in the 18th Century}

In the investigated register books of Kèdainiai municipal institutions of the 18th century, 115 naming instances of townswomen were found. In different naming types (with two, three or four members), first names were recorded first, and usually those were of Christian origin. In the historical documents of the 18th century, 23 canonical forms of Christian names were found, e.g.: 1760 Agata $\mathrm{KAK}_{10}$ 19; 1704 Agatha $\mathrm{KAK}_{3} 4 \mathrm{v} ; 1768$ Agaty gen. sg. $\mathrm{KAK}_{15} 45$. Comp. curt. n. Agotà (LVKŽ 62); 1704 Anna $\mathrm{KAK}_{3}$ 5; 1783 anna $\mathrm{KAK}_{21}$ 12; 1732 Anny gen. sg. $\mathrm{KAK}_{6}$ 10.Comp. curt. n. Onà (LVKŽ 294-295); 1778 Barbara KAK 19 68. Comp. curt. n. Barborà (LVKŽ 90); 1791 Brygida KAK $_{23}$ 21; 1750 Brygita KAK $_{8}$ 12. Comp. curt. n. Brigità (LVKŽ 101); 1782 Catharina $\mathrm{KAK}_{20}$ 127; 1788 Kataryna $\mathrm{KAK}_{21}$ 93; 1704 Katarzyna $\mathrm{KAK}_{3}$ 4; 1714 Katarzyna $\mathrm{KAK}_{3}$ 36; 1778 Katarzynny gen. sg. KAK 18 66; 1704 Katharzyna $\mathrm{KAK}_{3}$ 4. Comp. curt. n. Kotrynà (LVKŽ 235); 1704 Christina gen. sg. KAK 3 9. Comp. curt. n. Kristinà (LVKŽ 237); 1784 Elena $\mathrm{KAK}_{21}$ 23; 1798 Helena $\mathrm{KAK}_{23}$ 78. Comp. curt. n. Elenà (LVKŽ 146); 1794 Elisabeth $\mathrm{KAK}_{24}$ 4; 1777 Elisabetha $\mathrm{KAK}_{17}$ 220; 1720 Elizabiecie dat. sg. KAK 3 109; 1783

Elzbieta $\mathrm{KAK}_{21}$ 5. Comp. curt. n. Elzbietà (LVKŽ 148); 1780 Eva $\mathrm{KAK}_{20}$ 38; 1714 Ewa $\mathrm{KAK}_{3}$ 
Onomástica Desde América Latina, n.4, v.2, julho - dezembro, 2021, p. 4 -31. ISSN 2675-2719 https://doi.org/10.48075/odal.v0i0.27485

67. Comp. curt. n. Ievà (LVKŽ 195); 1706 Gertruda KAK $_{3}$ 12; 1774 Giertruda KAK $_{24}$ 1. Comp. curt. n. Gertrūdà (LVKŽ 176); 1762 Iudyta KAK 10 81; 1795 Judytty gen. sg. KAK 24 18. Comp. curt. n. Judità (LVKŽ212); 1778 Jadwiga KAK 19 43. Comp. curt. n. Jadvygà (LVKŽ 201); 1732 Justyney dat. sg. KAK5 2. Comp. curt. n. Justinà (LVKŽ 216); 1721 Klary gen. sg. KAK 4. Comp. curt. n. Klarà (LVKŽ 230); 1795 Konftancyi gen. sg. KAK 24 19v; 1798 Konstancyi gen. sg. $\mathrm{KAK}_{23}$ 87. Comp. curt. n. Konstáncija (LVKŽ 234); 1749 Magdalena $\mathrm{KAK}_{2}$ 124. Comp. curt. n. Magdalenà (LVKŽ 255-256); 1787 Matgorzata KAK 21 70v. Comp. curt. n. Margarità (LVKŽ 263); 1778 Mariana $\mathrm{KAK}_{19}$ 44v; 1704 Marianna $\mathrm{KAK}_{3}$ 3v; 1782 Mariianna $\mathrm{KAK}_{21}$ 3v; 1789 Marjany gen. sg. KAK 21 105. Comp. curt. n. Marijonà (LVKŽ 264); 1720 Regina $\mathrm{KAK}_{3}$ 99v. Comp. curt. n. Reginà (LVKŽ 311); 1785 Rosalie KAK 21 30; 1768 rozalia KAK 1545. Comp. curt. n. Rozãlija (LVKŽ 317); 1781 Teresie dat. sg. KAK 20 82; 1765 Tere/sa KAK 10115. Comp. curt. n. Terèsè (LVKŽ 347); 1761 Urszula KAK 10 10. Comp. curt. n. Uršùlè (LVKŽ 354); 1760 Zoffyi dat. sg. $\mathrm{KAK}_{10}$ 14; 1714 Zofia $\mathrm{KAK}_{3}$ 30; 1704 Zofyi gen. sg. $\mathrm{KAK}_{3}$ 4v; 1767 Zofija $\mathrm{KAK}_{15}$ 8; 1715 Zophia $\mathrm{KAK}_{3}$ 69; 1714 Zophia KAK 3 30. Comp. curt. n. Zòfija (LVKŽ $385)$.

Forty-eight $(41.74 \%)$ Kèdainiai townswomen were recorded by their first name and the name of the husband or father, e.g.: 1768 Pani Zofia Ciołkiewiczowa Obyw(atelka) Kieyd(anska) KAK $_{15}$ 45; 1776 Pani Magdaleny Jaruszewiczowey gen. sg. KAK 17 152; 1776 Pani Anna Ja/sułowiczowa Burmistrzowa $\mathrm{KAK}_{15}$ 75; 1761 Iudyty Mackiewiczowey gen. sg. KAK 10 4v; 1769 Pani Katarzyna Masandowiczowa Obyw(atelka) Kiey(danska) $\mathrm{KAK}_{15}$ 63; 1761 Iudyta Micewiczowa Obywatelk(a) Kieyd(anska) $\mathrm{KAK}_{10} 52 ; 1783$ Elzbieta Stefanowiczowa $\mathrm{KAK}_{21}$ 5; 1795 Judytty Tarafsewiczowey Obywatelki Miafta Kieydan gen. sg. $\mathrm{KAK}_{24}$ 18; 1772 Uczciwa Marianna Wandowska Obywatelka Kejdan(ska) $\mathrm{KAK}_{16}$ 66; 1749 Magdalena Wirbałowa $\mathrm{KAK}_{2}$ 
Onomástica Desde América Latina, n.4, v.2, julho - dezembro, 2021, p. 4 -31. ISSN 2675-2719 https://doi.org/10.48075/odal.v0i0.27485

124; 1762 Uczciwa Indyta Woyciechowiczowa Obywatelka Kieidan(ska) KAK 10 81; 1776 Uczciwey Barbarze Wołkowiczowey dat. sg. $\mathrm{KAK}_{17} 150$.

Those recordings contain some naming instance by the first name and personal name after the husband, with an attached indication "widow" in Polish wdowa, Lat. vidua, i.e., našle in Lithuanian, e.g.: 1773 Pani Barbarze Gorkowey wdowcie dat. sg. KAK 16 76; 1776 Uczciwa Maryanna Illewiczowa wdowa $\mathrm{KAK}_{17}$ 166; 1704 Wdowy Anny Jankowey gen. sg. KAK 3 5v; 1788 Uczciwa Pani Konstancya Jaruszewiczowa wdowa Obywatelka Miasta Kieydan $\mathrm{KAK}_{22}$ 135; 1794 Honefta Elisabeth Kardafzewiczowa vidua $\mathrm{KAK}_{24}$ 4; 1761 Uczciwa Anna Kurolowiczowa wdowa $\mathrm{KAK}_{10}$ 52; 1762 Uczciwa Anna Lubenowa wdowa Maystrowa Kunfztu Kowalskiego $\quad$ Ob(ywatelka) $\quad$ M(iasta) $\quad$ Kieydan $\mathrm{KAK}_{10} \quad 66 ; 1790 \quad<\ldots>$ Magdalena Matukiewiczowa wdowa Obywatelka Kieydan(ska) KAK 21 116v; 1788 Uczciwa P(ani) Ewa Razulewiczowa wdowa Obyw(atelka) Kieydan(ska) $\mathrm{KAK}_{22} 131$.

Just as in inscriptions of the 17th century, fewer recordings of young women after the father were noticed, e.g.: 1796 Ucz(ciwe)y Gertrudy Gycowny Obyw(atelki) gen. sg. KAK 24 77; 1776 Anna Illewiczowna Obyw(atelka) Kieydan(ska) KAK 17 166; 1704 Anna Jankowna $\mathrm{KAK}_{3}$ 5; 1704 Katharzyna Klementowiczowna abl. sg. $\mathrm{KAK}_{3} 3 ; 1704$ Agatha Kordaszewiczowna $\mathrm{KAK}_{3}$ 4v; 1704 Christina Paszkiewiczowna KAK 37v; 1709 Elzbieta Slizewiczowna KAK 1 100; 1721 Klary Szymkiewiczowney gen. sg. KAK 4 4; 1704 Catharince Waskiewiczowny gen. sg. KAK 3 5v.

Names of townspeople containing Polish suffixes of the -sk-type may be considered as the ending $-a$ derivatives, e.g.: 1732 Justynie Dubinskiey dat. sg. $\mathrm{KAK}_{5} 2 ; 1768$ Krystyna Skaczkowska KAK 15 36; 1704 Marianna Szylinska KAK 3 4; 1765 Usciwey Barbarze Uscinowskiey dat. sg. KAK 10 140; 1775 Uczciwa Dorota Wabzdowska wdowa Obyw(atelka) 
Onomástica Desde América Latina, n.4, v.2, julho - dezembro, 2021, p. 4 -31. ISSN 2675-2719 https://doi.org/10.48075/odal.v0i0.27485

Kieydan(ska) KAK 17 98; 1772 Uczciwa Marianna Wandowska Obywatelka $\mathrm{KAK}_{16}$ 66; 1761 $<\ldots>$ y samey Mariannie Wasilewskiey dat. sg. $\mathrm{KAK}_{1} 162$.

Other naming types were less productive. Four personal names were used in the recordings of $22(19.13 \%)$ women, e.g.: 1716 Uczciwey Jadwidze Dilewiczownie Janowey Augutowiczowey dat. sg. KAK 3 76v; 1778 Katarzyny Sokołowczanki Janowey Golniewskiey gen. sg. $\mathrm{KAK}_{1}$ 98; 1778 Annie Tylidayczance Macieiowey Golniewskiey dat. sg. KAK 1 98; 1704 Katarzyna Kordaszewiczowna Stanistawowa Hermanowiczowa $\mathrm{KAK}_{3} 4 ; 1714$ Ia Ewa Jozephowiczowna Kazimierzowna Jerczewska $\mathrm{KAK}_{3}$ 67; 1720 Uczciwey Anny Waskiewiczowny Kazimierzowey Juchniewiczowey Obywatelki Miasta Kieydan KAK $103 ; 1716$ Ucziwey Agatcie

Thomaszewskiey Kazimierzowey Kapłayciowey dat. sg. $\mathrm{KAK}_{1} 78 ; 1708$ Ja Katharzyna Komontowna Jakubowa Karpowska $\mathrm{KAK}_{3} 14 \mathrm{v} ; 1704$ Pani Dorocie Adamowiczownie Stephanowey Kordaszewiczowey dat. sg. $\mathrm{KAK}_{3}$ 4v; 1704 Katharzynie Waskiewiczownie Adamowey Laucewiczowey dat. sg. $\mathrm{KAK}_{3}$ 5; 1714 Ia Zofia Gieniowna Jer3yna Mackiewiczowa $\mathrm{KAK}_{3} 30 ; 1704$ Mariannie Szyliszczance Franciszkowey Matulaytysowey dat. sg. KAK 3 3v; 1720 Ja Apolonia Pafzkiewiczowna Jozephowa Monckwilowa Obywatelka Kieydanska $\mathrm{KAK}_{2}$ 56; 1714 Katarzyna Stanilewiczowna Mathiaszowa Moszkiewiczowa KAK 3 36; 1721 Helenna Dyczpinigayciowna Kazimierzowa Narmunfka <...> $\mathrm{KAK}_{2}$ 58; 1720 Ja Regina Tylkinowiczowna Janowa Rymdeykowa $\mathrm{KAK}_{3}$ 99v; 1720 Pani Elizabiecie Dulayciownie Piotrowey Skirgayliney dat. sg. $\mathrm{KAK}_{3}$ 109; 1720 Paniey Anni Federowiczownie Krzysztophowey Szeybowey dat. sg. $\mathrm{KAK}_{3}$ 100; 1704 Zofia Kordaszewiczowna Jakubowa Szymayciowa KAK 3 4; 1714 Uczciwey Katharzynie Woszkiewiczownie Gerzyney Walentynowiczowey dat. sg. $\mathrm{KAK}_{3}$ 58; 1716 Uczciwey Mariannie Lubeynownie Kazimierzowey Wencewiczowey dat. sg. $\mathrm{KAK}_{3} 80 \mathrm{v}$. 
Onomástica Desde América Latina, n.4, v.2, julho - dezembro, 2021, p. 4 -31. ISSN 2675-2719 https://doi.org/10.48075/odal.v0i0.27485

The town register books contain $17(14.79 \%)$ recordings of women without the first name, giving only two personal names made from the name of the husband and the second anthroponym. The first name of women is not given in these recordings as the most important are the suffixal anthroponyms indicating the belonging to the husband. In the opinion of clerks, male individuals were clearly more important because of their economic and social status. The men were called their wives' guardians, proxies and representatives in the Kèdainiai City Magistrate's Court. According to the laws of that time, men represented not only their wives in the court, but also mothers, sisters and daughters (Ragauskaite 2005: 33, 143). For these reasons the first names of women were just not given in these instances, e.g.: 1704 Abrahamowa Adamowiczowa $\mathrm{KAK}_{3}$ 4v; 1770 Michałowa Gaudziewiczowa $\mathrm{KAK}_{15}$ 113; 1733 Piotrowa Ilewiczowa abl. sg. $\mathrm{KAK}_{5} 21 ; 1762<\ldots>$ Francifzkowa Jakowiczowa $\mathrm{KAK}_{15}$ 65; 1774 Stawetna Pani Jozefowa Jankowska Obywatelka Kieydan(ska) KAK $_{17}$ 31; 1764 Kazimierzowa Kardafzewiczowa $\mathrm{KAK}_{10}$ 87; 1732 Ja Michałowa Macewiczowa Obywatelka Kieydanska $\mathrm{KAK}_{2}$ 82; 1704 Krzysztofowa Orlikowa KAK $_{3}$ 4v; 1770 Kazimierzowa Skaczkowska KAK $_{15}$ 113; 1704 Stanisławowa Waskiewiczowa $\mathrm{KAK}_{3}$ 5; 1725 Ucfciwa Jakubowa Wirbałowa $\mathrm{KAK}_{4}$ 118v. A similar type of naming is comprised of a personal name indicating the belonging and the appellation "widow" našle in Lithuanian (wdowa in written Polish sources), e.g.: 1781 Uczciwey Tomaszowey Illewiczowey wdowy Obyw(atelki) gen. sg. KAK 20 103; 1764 Szymonowa Kiełpszowa wdowa $\mathrm{KAK}_{10}$ 87; 1787 Uczciwa P(ani) Stanisławowa Razulewiczowa wdowa Obyw(atelka) Kieydan(ska) $\mathrm{KAK}_{22}$ 123; 1709 Kasprowa Snitkanska wdowa $\mathrm{KAK}_{1} 100$.

$15(13.04 \%)$ townspeople were recorded using only one anthroponym (a suffixal personal name), e.g.: 1759 Ucziwey Pani Augutowiczowey Obywat(elki) Kieyd(anskiei) gen. sg. KAK 10 1; 1766 Stawetna Pani Ciołkiewiczowa KAK 10 17; 1784 Giedeykowa KAK 21 17v; 1760 
Onomástica Desde América Latina, n.4, v.2, julho - dezembro, 2021, p. 4 -31. ISSN 2675-2719 https://doi.org/10.48075/odal.v0i0.27485

Iurafzewiczowa $\mathrm{KAK}_{10}$ 23; 1777 Jasułowiczowa abl. sg. KAK 19 9; 1796 Kasprowska $\mathrm{KAK}_{24}$ 94v; 1761 Lubenowa $\mathrm{KAK}_{10}$ 34; 1784 Mackiewiczowa $\mathrm{KAK}_{21}$ 17v; 1760 Narmunska $\mathrm{KAK}_{10}$ 23; 1798 Rymowiczowey gen. sg. $\mathrm{KAK}_{23}$ 62; 1770 Szarłowska $\mathrm{KAK}_{15}$ 99; 1777 Szczepowiczowa $\mathrm{KAK}_{19}$ 9; 1794 Szpilewiczowey gen. sg. KAK 24 7v; 1760 Wabzdowska $\mathrm{KAK}_{10} 23$.

The 13 remaining $(11.30 \%)$ names were recorded using three anthroponyms, e.g.: 1760 Uczciwa Agata Symonowa Amalewiczowa $\mathrm{KAK}_{10}$ 19; 1761 Heleny Wawrzyncowey Iasułowiczowey gen. sg. $\mathrm{KAK}_{10}$ 56; 1710 Katerzyny Jafiulewiczowny Illewiczowey gen. sg. $\mathrm{KAK}_{2}$ 53v; 1714 honesta Zophia Gieniowna Mackiewiczowa KAK 3 30; 1732 Marrynie Eygirdownie Narmunskiey dat. sg. KAK5 1; 1714 Paniey Judytcie Adgajownie Rudzianskiey dat. sg. $\mathrm{KAK}_{3}$ 36v; 1761 Zofyi Michatowey Szwaykowskiey gen. sg. KAK 10 40; 1736 Barbara Dylayciowna Uscinowska $\mathrm{KAK}_{5}$ 64; 1705 Magdalena Piotrowa Wandziewska KAK 3 8v; 1706 Gertruda Stefanowa Zelwiowa <...> Obywatelka Miasta Kieydan $\mathrm{KAK}_{3} 12$.

The surnames of clerks responsible for the researched documentation are known and their etymology allows to believe that most of them were Polish (RAGAUSKAITE், 2016b: 112). The nationality of the clerks and their individual preferences could have influenced the methods of Polonization of Lithuanian anthroponyms. These extralinguistic circumstances had an effect on the writing of Lithuanian historical personal names of Kèdainiai residents. Therefore, their solutions to the recording of female anthroponyms and their forms were different. Most anthroponyms were modified in a variety of ways. This was also impacted by linguistic reasons, and especially the interplay between Lithuanian and Polish languages. The existing data demonstrate the tendency of the 17 th-18th centuries to augment anthroponyms of Kèdainiai townspeople by adding Slavic suffices -ova and -ovna, e.g.: Uczciwa Helena Giedeykowa wdowa Obyw(atelka) Kieydan(ska) $\mathrm{KAK}_{23}$ 78; 1767 Utsciwey Anny Grygalunowey wdowy gen. sg. 
Onomástica Desde América Latina, n.4, v.2, julho - dezembro, 2021, p. 4 -31. ISSN 2675-2719 https://doi.org/10.48075/odal.v0i0.27485

$\mathrm{KAK}_{15}$ 32; 1768 Pani Katarzyna Gulbinowa wdowa Obyw(atelka) Kieyd(anska) $\mathrm{KAK}_{15}$ 43;

1762 Uczciwa Anna Lubenowa wdowa Maystrowa Kunfztu Kowalskiego KAK 10 66; 1780 Uczciwey Katarzyny Rymszewiczowey wdowy Obyw(atelki) Kieydanskiei gen. sg. $\mathrm{KAK}_{20}$ 79; 1761 Helena Treygiowa Obywat(elka) Kieyd(anska) $\mathrm{KAK}_{10} 50$.

Kèdainiai register books of the 18th century predominantly contain surnames of townspeople with Slavic suffixes -ova, -ovna and Polish suffixes of the -sk- type. However, single cases of surnames with Lithuanian endings or suffixes could still be found during this period as well, e.g.: *Mažaičia: $1731<\ldots>$ Ja Zofia Mazaycia Zwilgowa $\mathrm{KAK}_{2}$ 74. Regarding maž- comp. curt. sur. Mažéika, Mažeĩtis ir kt. (LPŽ II 191); *Meškaičia: 1704 z Paniq Jadwiga Marcinowiczowna Augustynowiczowna alias Mieszkaycia $\mathrm{KAK}_{3}$ 4v. Comp. curt. sur. Meškà (LPŽ II 212);

In this context of the investigation into the naming Kèdainiai women of the 18th century, it seems imperative to define the dissemination of female personal names with Lithuanian suffixes in different types of historical documents. Such historical anthroponymic data of townspeople, their use and inventory help to understand the extent of Slavisation used for female personal names as well as the situation of the Lithuanian language in a town of the times (KIAUPA, 2000: 87-88). It has been observed that Slavisation had a different effect on the naming of townspeople in Kèdainiai register books and parish registers. This can be demonstrated based on the 1752-1799 register of christenings kept by the St. George's Church of Kèdainiai Parish. This Catholic parish church provided christenings for children of Kèdainiai townswomen and women from the surrounding villages. Mostly, the women were Lithuanian as their names were recorded using Lithuanian suffixes: -iene (written as: -enie), -aičia (-ayczia) and -yčia (-iczia). For comparison, only those 26 names of mothers and Godmothers of 
Onomástica Desde América Latina, n.4, v.2, julho - dezembro, 2021, p. 4 -31. ISSN 2675-2719 https://doi.org/10.48075/odal.v0i0.27485

christened infants were selected that had an indication Keydany, e.g.: *Lukošaitiené: 1761 Anna Eukoszaytenie <...> Keydany KKK120. Comp. curt. sur. Lukošáitis (LPŽ II 114); *Augustaičia: 1755 Agatha Avgustayczia <...> Keydany KKK27. Comp. curt. sur. Aügustas, Augùstas, Augustas (LPŽ I 137); *B u ša i čia: 1756 Agatha Buszayczia <...> Keydany KKK 38. Comp. curt. sur. Bùšas (LPŽ I 362-363); * Čiužaičia: 1765 Marianna Cuzayczia <...> Keydany KKK190. Comp. curt. sur. Čiùžas (LPŽ I 436); *Do minaičia: 1766 Marianna Dominayczia <...> Keydany KKK 201. Comp. curt. sur. Dõminas, Domìnas, Dominas (LPŽ I 513); *Griškaičia: 1754 Magdalena Gryszkayczia <...> Keydany KKK18. Comp. curt. sur. Griškà (LPŽ I 721); *Gu s ta ič i a : 1758 Magdalena Gustayczia <...> Keydany KKK 69. Comp. curt. sur. Gùstas (LPŽ I 748); *Jakštaičia: 1768 Dorothea Jaksztayczia <...> Keydany KKK226. Comp. curt. sur. Jãkštas (LPŽ I 789); *Jukaǐ̌ia: 1758 Anna Jukayczia <...> Keydany KKK65. Dèl juk- comp. curt. sur. Jùkelis, Jukevičius (LPŽ I 852); *Ki r š a i č i a : 1757 Anna Kirszayczia $\quad<. .>$ Keydany KKK 68. Comp. curt. sur. Kìřsas (LPŽ I 1002); *Matušaitaičia: 1754 Zophia Matusaytayczia <...> Keydany KKK 44. Comp. curt. sur. Matušáitis (LPŽ II 184); *Mikštaičia: 1753 Anna Miksztayczia <...> Keydany KKK10. Comp. curt. sur. Mikštas (LPŽ II 233); *Ne me ikai čia: 1788 Catharina Niemeykayczia <...> Keydany KKK413. Comp. curt. sur. Neméika, Nemeikà (LPŽ II 318); *Olš evs kaičia: 1752 Anna Olszewskayczia <...> Keydany KKK4. Comp. curt. sur. Olšèvskas (LPŽ II 348); *Petrilaitaičia: 1758 Elisabeth Petrytaytayczia <...> Keydany KKK 66. Comp. curt. sur. Petrilaitis (LPŽ II 441); *Pupkaičia: 1760 Helena Pupkayczia <...> Keydany KKK105. Comp. curt. sur. Pupkà (LPŽ II 536); *Pǔ̌ynaičia: 1788 Marianna Puszynayczia <...> Keydany KKK413. Comp. curt. sur. Pùšynas, Pušýnas (LPŽ II 541); *Soraičia: 1756 Magdalena Sorayczia $<\ldots>$ Keydany KKK37. Comp. curt. sur. Sóra (LPŽ II 779); 
Onomástica Desde América Latina, n.4, v.2, julho - dezembro, 2021, p. 4 -31. ISSN 2675-2719 https://doi.org/10.48075/odal.v0i0.27485

*Stašaitaičia: 1761 Juditha Staszaytayczia <...> Keydany KKK118. Comp. curt. sur. Stašáitis (LPŽ II 810); *Šniraičia: 1760 Anna Sznirayczia <...> Keydany KKK 100. Comp. curt. sur. Šniras (LPŽ II 966); *Trumpaičia: 1765 Anna Trumpayczia <...> Keydany KKK188. Comp. curt. sur. Trùmpa (LPŽ II 1073); *Užkuraitaičia: 1762 Catharina

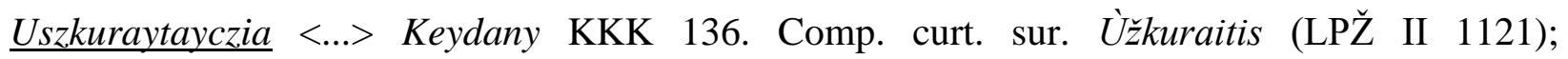
*Valiūnaičia: 1765 Magdalena Walunayczia <...> Keydany KKK185. Comp. curt. sur. Valiūnas (LPŽ II 1154); *Vir b a la i č i a : 1755 Catharina Wirbałayczia <...> Keydany KKK30. Comp. curt. sur. Virbalas (LPŽ II 1227); *Višnevskaič ia : 1752 Marianna Wisniewskayczia <...> Keydany KKK4. Comp. curt. sur. Višnèvskas (LPŽ II 1237); *Rasakyčia: 1754 Catharina Rasakiczia <...> Keydany KKK13. Dèl rasak- comp. curt. sur. Rasakãckas, Rasakauskas (LPŽ II 575).

Kèdainiai Reformists of the 17th-18th centuries fostered the Lithuanian language. In the Grand Duchy of Lithuania of the 17th century, the town of Kedainiai was the Reformist centre of Lithuanianness. In this town, the Mass was celebrated in Lithuanian starting with the third decade of the 17th century and, with a five-year interruption (1682-1687), continued until 1785 (LUKŠAITĖ, 1970: 15, 23). Town burgomaster Steponas Jaugelis Telega personally participated in the session of town Reformists, which decided to organise books required for the Mass in Lithuanian. The synod was responsible for appointing priests who knew Lithuanian for full-time service in Kèdainiai (LUKŠAITĖ, 2001: 10). The book Knyga nobažnystès (The Book of the Christian Faith) was published in Kèdainiai, not in Vilnius. According to Lukšaite (LUKŠAITĖ, 2001: 4), this was the biggest Lithuanian publication of the century, which accommodated four books under one title; it was a sign that over the first half of the 17th century, the community of the Lithuanian Reformed church - Unitas Lithuaniae (The Evangelical Reformed Church of 
Onomástica Desde América Latina, n.4, v.2, julho - dezembro, 2021, p. 4 -31. ISSN 2675-2719 https://doi.org/10.48075/odal.v0i0.27485

Lithuania) - had experienced significant shiftsin its cultural self-awareness. These circumstances clearly demonstrate that the Lithuanian language was used in the public life of Kedainiai in the 17th-18th centuries. These manifestations of Lithuanianness were also somewhat reflected in the analysed manuscripts.

\section{Conclusions}

The development of naming residents of the town was complicated and had a distinct specificity in different centuries. This was impacted by particularities of legal administrative sources of Kedainiai, the context of registers and clerks charged with the registration of court cases of the time. In the 17th century, women were mostly recorded using four anthroponyms, and in the 18th century, only two personal names (i.e., first name and surname after the father or husband) were used. Canonical Christian names were found in different recordings.

Results of the investigation into the historical anthroponymy of Kedainiai demonstrated that personal names of the seventeenth century were Polonised: cases of phonetic substitution were noted; some Lithuanian patronymic suffixes were replaced with Slavic suffixes -ova, -ovna. The most extensive Polonisation of anthroponyms occurred in collections of Kèdainiai registers of the 18th century. In this period, Slavic patronymic suffixes -ova, -ovna and suffixes of -sktype were predominant in surnames of Kèdainiai women.

Polonisation had a different effect on the naming of women in Kèdainiai register books of the 18th century and the 1752-1799 baptismal register kept by Kèdainiai Parish. This church source contains more female surnames with Lithuanian suffixes. It leads to the conclusion that female personal names bearing Lithuanian suffixes possibly were also recorded in the manuscripts of other cities. 
Onomástica Desde América Latina, n.4, v.2, julho - dezembro, 2021, p. 4 -31. ISSN 2675-2719

https://doi.org/10.48075/odal.v0i0.27485

\section{Appendix}

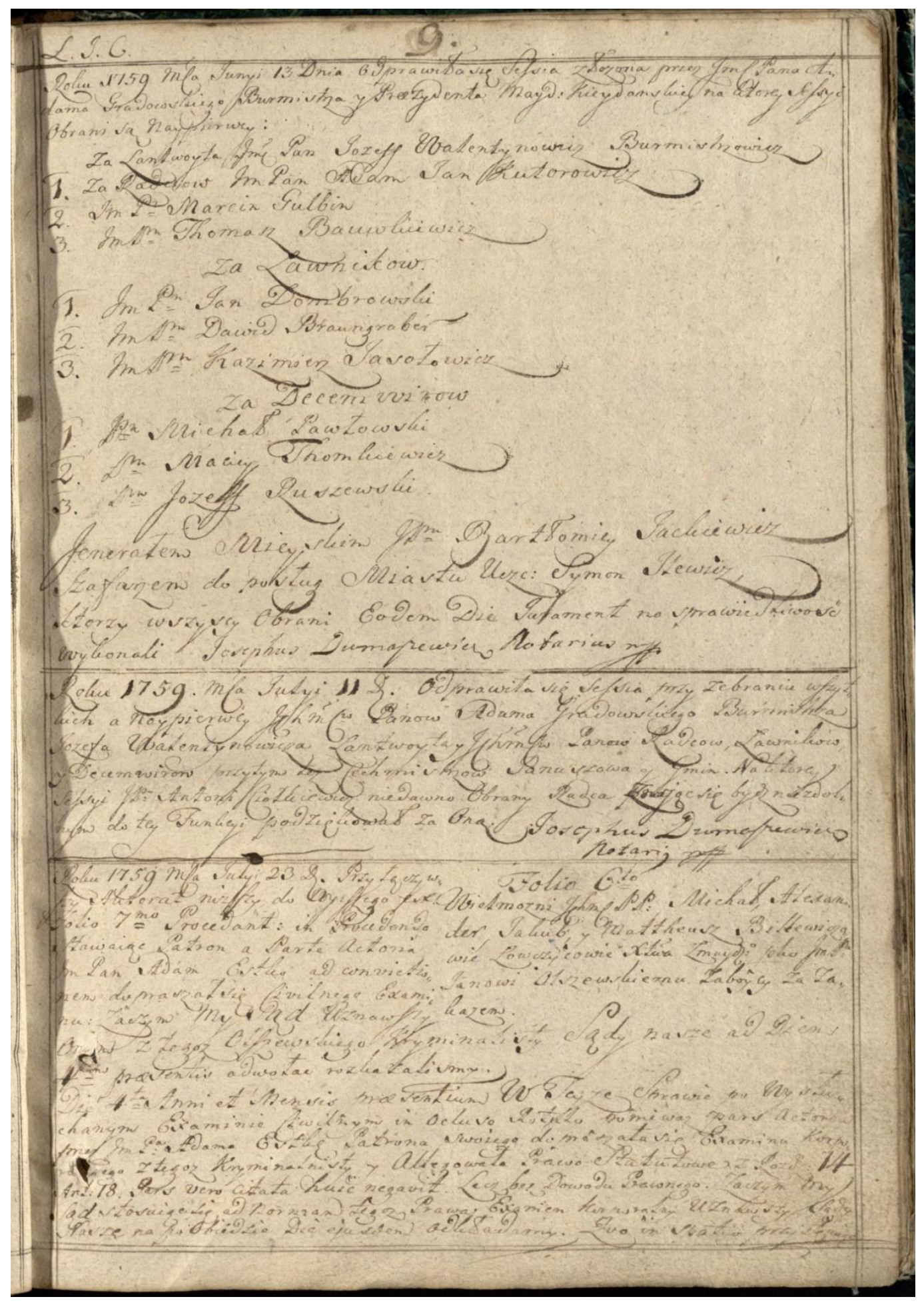

Figure 1: Kèdainiai register book of 1750-1766, fragment dated 1759 (p. 9) 
Onomástica Desde América Latina, n.4, v.2, julho - dezembro, 2021, p. 4 -31. ISSN 2675-2719

https://doi.org/10.48075/odal.v0i0.27485

Recebido em 31/05/2021

Aceito em 08/07/2021

Publicado em em 25/08/2021

\section{References}

\section{Archival Sources}

$\mathrm{KAK}_{1}$ - Kèdainių 1623-1806 m. aktų knyga [Kèdainiai register book of 1623-1806]. Lietuvos valstybès istorijos archyvas [Office of the Chief Archivist of Lithuania], hereafter - LVIA, f. SA, b. 15360 .

$\mathrm{KAK}_{2}$ - Kèdainių aktų 1690-1775 m. knyga [Kèdainiai register book of 1690-1775]. LVIA, f. SA, b. 15357.

$\mathrm{KAK}_{3}-$ Kèdainių aktų 1704-1720 m. knyga [Kèdainiai register book of 1704-1720]. LVIA, f. SA, b. 15358.

$\mathrm{KAK}_{4}$ - Kèdainių aktų 1721-1725 m. knyga [Kèdainiai register book of 1721-1725]. LVIA, f. SA, b. 15359.

$\mathrm{KAK}_{5}-$ Kèdainių aktų 1732-1740 m. knyga [Kèdainiai register book of 1732-1740]. LVIA, f. SA, b. 15361.

$\mathrm{KAK}_{6}-$ Kèdainių aktų 1732-1743 m. knyga [Kèdainiai register book of 1732-1743]. LVIA, f. SA, b. 15362.

$\mathrm{KAK}_{7}-$ Kèdainių aktų 1744-1750 m. knyga [Kèdainiai register book of 1744-1750]. LVIA, f. SA, b. 15363. 
Onomástica Desde América Latina, n.4, v.2, julho - dezembro, 2021, p. 4 -31. ISSN 2675-2719 https://doi.org/10.48075/odal.v0i0.27485

$\mathrm{KAK}_{8}-$ Kèdainių aktų 1750-1752 m. knyga [Kèdainiai register book of 1750-1752]. LVIA, f. SA, b. 15364.

$\mathrm{KAK}_{9}-$ Kèdainių aktų 1750-1761 m. knyga [Kèdainiai register book of 1750-1761]. LVIA. f. SA, b. 15367.

KAK $_{10}-$ Kèdainių aktų 1750-1766 m. knyga [Kèdainiai register book of 1750-1766]. LVIA. f. SA, b. 15368.

$\mathrm{KAK}_{11}$ - Kèdainių aktų 1753-1755 m. knyga [Kèdainiai register book of 1753-1755]. LVIA. f. SA, b. 15365 .

$\mathrm{KAK}_{12}$ - Kèdainių aktų 1756-1758 m. knyga [Kèdainiai register book of 1756-1758]. LVIA. f. SA, b. 15366.

$\mathrm{KAK}_{13}$ - Kèdainių aktų 1762-1763 m. knyga [Kèdainiai register book of 1762-1763]. LVIA, f. SA, b. 15369.

$\mathrm{KAK}_{14}$ - Kèdainių aktų 1763-1770 m. knyga [Kèdainiai register book of 1763-1770]. LVIA, f. SA, b. 15370.

KAK $_{15}$ - Kèdainių aktų 1767-1770 m. knyga [Kèdainiai register book of 1767-1770]. LVIA, f. SA, b. 15371 .

KAK $_{16}$ - Kèdainių aktų 1771-1773 m. knyga [Kèdainiai register book of 1771-1773]. LVIA, f. SA, b. 15372 .

$\mathrm{KAK}_{17}$ - Kèdainių aktų 1774-1777 m. knyga [Kèdainiai register book of 1774-1777]. LVIA, f. SA, b. 15373. 
Onomástica Desde América Latina, n.4, v.2, julho - dezembro, 2021, p. 4 -31. ISSN 2675-2719 https://doi.org/10.48075/odal.v0i0.27485

$\mathrm{KAK}_{18}$ - Kèdainių aktų 1777-1779 m. knyga [Kèdainiai register book of 1777-1779]. LVIA, f. SA, b. 15374.

$\mathrm{KAK}_{19}$ - Kèdainių aktų 1777-1780 m. knyga [Kèdainiai register book of 1777-1780]. LVIA, f. SA, b. 15375 .

$\mathrm{KAK}_{20}$ - Kèdainių aktų 1779-1782 m. knyga [Kèdainiai register book of 1779-1782]. LVIA, f. SA, b. 15376.

$\mathrm{KAK}_{21}$ - Kèdainių aktų 1782-1791 m. knyga [Kèdainiai register book of 1782-1791]. LVIA, f. SA, b. 15377.

$\mathrm{KAK}_{22}$ - Kèdainių aktų 1783-1792 m. knyga [Kèdainiai register book of 1783-1792]. LVIA, f. SA, b. 15378.

$\mathrm{KAK}_{23}$ - Kèdainių aktų 1791-1806 m. knyga [Kèdainiai register book of 1791-1806]. LVIA, f. SA, b.15379.

KAK $_{24}$ - Kèdainių aktų 1794-1798 m. knyga [Kèdainiai register book of 1794-1798]. LVIA, f. SA, b. 15380.

KKK - Kèdainių Šv. Jurgio bažnyčios krikšto 1752-1799 m. metrikų knyga [1752-1799 baptismal register kept by the St. George's Church of Kedainiai Parish]. LVIA, f. 1424, ap. 1, b. 1 .

\section{Bibliography}


Onomástica Desde América Latina, n.4, v.2, julho - dezembro, 2021, p. 4 -31. ISSN 2675-2719 https://doi.org/10.48075/odal.v0i0.27485

Amaral, E. T. R. \& Seide, M. S. (2020). Nomes próprios de pessoa: introdução à antroponímia

brasileira. Blucher: São Paulo.https://openaccess.blucher.com.br/article-list/9786555500011$\underline{453 / \text { list\#undefined }}$

Jablonskis, K. (1979). Istorija ir jos šaltiniai. Vilnius: Mokslas.

Kiaupa, Z. (2000). Kauno vaito XVI a. vidurio knygos prabyla lietuviškai. In Z. Kiaupa et al., Kultūru sankirtos (pp. 66-92). Vilnius: Diemedžio leidykla.

Lepszy, K. (1953). Instrukcja wydawnicza dla źródel historycznych od XVI do połowy XIX wieku. Wrocław: Zakład narodowy im. Ossolińskich. Wydawnictwo Polskiej Akademii Nauk.

LPŽ I - Maciejauskienè, V.; Razmukaitè, M. \& Vanagas, A. (Ed.) (1985). Lietuviu pavardžiu žodynas (Vol. 1). Vilnius: Mokslas.

LPŽ II - Maciejauskienė, V.; Razmukaitè, M. \& Vanagas, A. (Ed.) (1989). Lietuviu pavardžiu žodynas (Vol. 2). Vilnius: Mokslas.

Lukšaitè, I. (1970). Lietuviu kalba reformaciniame judejime XVII a. Vilnius: Lietuvos TSR Mokslų akademijos Istorijos institutas.

Lukšaitė, I. (2001). Knygos nobažnystès (1653) parengimo kultūrinė aplinka. In I. Lukšaite et al., „Knyga nobažnystès krikščioniškos“ (1653) - XVII a. Lietuvos kultūros paminklas (pp. 4-28). Kèdainiai: Kèdainių krašto muziejus.

LVKŽ - Kuzavinis, K., \& Savukynas, B. (1994). Lietuviu vardu kilmés žodynas. Vilnius: Mokslo ir enciklopedijų leidykla.

Maciejauskienė, V. (1991). Lietuviu pavardžiu susidarymas XIII-XVIII a. Vilnius: Mokslas. 
Onomástica Desde América Latina, n.4, v.2, julho - dezembro, 2021, p. 4 -31. ISSN 2675-2719 https://doi.org/10.48075/odal.v0i0.27485

Maciejauskienè, V. (1994). Lietuviu pavardès: raida ir kilmé. Vilnius: Lietuvių kalbos institutas.

Ragauskaitė, A. (1999). XVI a. II pusès Kauno miestiečių lietuviškos kilmès asmenvardžiai. Acta Linguistica Lithuanica / Lietuvių kalbotyros klausimai, 41, 145-158.

Ragauskaitè, A. (2000). XVII a. pirmosios pusės Kauno miestiečių asmenvardžių kilmè. Acta Linguistica Lithuanica / Lietuvių kalbotyros klausimai, 43, 93-110.

Ragauskaitè, A. (2001). Kauno miestiečių pavardžių formavimasis XVI-XVIII a. Acta Linguistica Lithuanica / Lietuviu kalbotyros klausimai, 45, 123-143.

Ragauskaitė, A. (2002). XVII-XVIII amžių kauniečių (vyrų) pavardžių variantai. Acta Linguistica Lithuanica / Lietuviu kalbotyros klausimai, 47, 31-40.

Ragauskaitè, A. (2003). XVI-XVIII a. kauniečių moterų asmenvardžių daryba. Vārds un tā pētīšanas aspekti: Rakstu krājums, 7, 82-92.

Ragauskaitė, A. (2004). XVI-XVII a. joniškiečių asmenvardžiai. In J. Švambarytė-Valužienė (Ed.), Istoriniai tekstai ir vietos kultūra (pp. 8-18). Šiauliai, Ryga: Lucilijus.

Ragauskaitè, A. (2005). XVI-XVIII a. kauniečiu asmenvardžiai. Vilnius: Lietuvių kalbos instituto leidykla.

Ragauskaitė, A. (2006). Vietovardinès kilmès asmenvardžiai ir prievardžiai XVI a. Kauno aktų knygose. Baltu filoloǵija, 15(1-2), 81-94.

Ragauskaite, A. (2008). Tendencies in the evolution of hereditary surnames of townspeople in Lithuania from sixteenth to eighteenth centuries. Zunamen / Surnames, 3, 29-37. 
Onomástica Desde América Latina, n.4, v.2, julho - dezembro, 2021, p. 4 -31. ISSN 2675-2719 https://doi.org/10.48075/odal.v0i0.27485

Ragauskaite, A. (2009). Kada susidarè kauniečiu pavardès? In A. Judžentis (Ed.), Kalba ir žmonès (pp. 226-232). Vilnius: Vilniaus universiteto leidykla.

Ragauskaité, A. (2014). Kauno moterų asmenvardžiai XVI-XVII a. lietuvių istorinès antroponimijos kontekste. Kauno istorijos metraštis, 14, 7-17.

Ragauskaite, A. (2015). Seniausios Lietuvoje 1599-1621 metų Joniškio krikšto metrikų knygos joniškiečių moterų asmenvardžiai lietuvių istorinès antroponimijos kontekste. Acta Linguistica Lithuanica,73, 55-81.

Ragauskaitè, A. (2016a). Škotų pavardžių užrašymo tendencijos XVII-XVIII a. Kėdainių miesto istorijos šaltiniuose. Acta Linguistica Lithuanica, 75, 45-67.

Ragauskaité, A. (2016b). Germaniškos kilmės kėdainiečių antroponimai XVII-XVIII a. Kèdainių miesto istorijos šaltiniuose. Baltu filoloǵija, 25(2), 111-128.

Ragauskaitè, A. (2017). Kèdainiečių (lietuvių, lenkų) pavardžių formavimosi tendencijos XVIIXVIII amžiuje. Acta Linguistica Lithuanica, 77, 53-77.

Ragauskaite, A. (2018a). The patterns of recording the names of German residents in Kedainiai in the nineteenth century: Introductory remarks. Acta Baltico-Slavica, 42, 173-188. DOI: $\underline{10.11649 / \mathrm{abs} .2018 .009}$

Ragauskaitė, A. (2018b). XVII a. kėdainiečių lietuviškos kilmès asmenvardžiai. Acta Linguistica Lithuanica,78, 53-75.

Ragauskaitè, A. (2018c). XVII a. kẻdainiečiu asmenvardžių darybos tendencijos. Baltu filologija, 27, 101-118. DOI: http://doi.org/10.22364/bf.27.04 
Onomástica Desde América Latina, n.4, v.2, julho - dezembro, 2021, p. 4 -31. ISSN 2675-2719 https://doi.org/10.48075/odal.v0i0.27485

Ragauskaite, A. (2019). Joniškiečių vyrų asmenvardžių darybos tendencijos seniausioje Lietuvoje 1599-1621 m. Joniškio krikšto metrikų knygoje. Acta Linguistica Lithuanica, 80, 177194. DOI: https://doi.org/10.35321/all80-08

Ragauskaite, A. (2021). Tendencies of formation of anthroponyms of rural residents in the oldest Lithuanian parish register of Joniškis dated 1599-1621. Onomástica desde América Latina, 2(3), 2-29. DOI: https://doi.org/10.48075/odal.v0i0.25781

Seide, M. S. \& Petrulione, L. (2018). Between Languages and Cultures: an Exploratory Comparative Study of Usage of Lithuanian and Brazilian Masculine Anthroponyms. Revista de estudos da Linguagem, 26, 1201-1226.

http://www.periodicos.letras.ufmg.br/index.php/relin/article/view/12745

Seide, M. S. \& Petrulionè, L. (2020). Formation and usage of hypocoristic forms in Brazilian, Portuguese and Lithuanian. Revista Alfa, 64(1), 1-27.

https://periodicos.fclar.unesp.br/alfa/article/view/11611/9330

Šinkūnas, P. (1928). Kèdainiu miesto istorija. Kaunas: „Spindulio“ B-vès spaustuvè.

Tyla, A. (2000). Decemviratas arba Tertio ordo communitatis Kèdainiu savivaldoje. Lietuvos istorijos metraštis 1999. Vilnius: Lietuvos istorijos institutas, 64-76.

Tyla, A. (2002). Lietuvos magdeburginiu miestu privilegijos ir aktai: Kédainiai, A. Tyla, R. Firkovičius \& D. Antanavičius (Eds.). Vilnius: Lietuvos istorijos instituto leidykla.

Tworek, S. (1966). Z dziejów kalwinizmu w Wielkim Księstwie Litewskim w XVIII w. Annales Universitatis Mariae Curie-Skłodowska, 21(8), 193-202. 
Onomástica Desde América Latina, n.4, v.2, julho - dezembro, 2021, p. 4 -31. ISSN 2675-2719 https://doi.org/10.48075/odal.v0i0.27485

Tworek, S. (1969). Materiały do dziejów kalwinizmu w Wielkim Księstwie Litewskim w XVII wieku. Odrodzenie i reformacja w Polsce, 14, 213-215.

Wolff, A. (1957). Projekt instrukcji wydawniczej dla pisanych źródeł historycznych do połowy XVI wieku. Studia źródtoznawcze, 1, 155-181.

Žirgulis, R. (2002a). Three Hundred Years of Multiculturalism in Kèdainiai. In G. Potašenko (Ed.), The Peoples of the Grand Duchy of Lithuania (pp. 130-140). Vilnius: Aidai.

Žirgulis, R. (2002b). Škotų bendruomenè Kėdainiuose 1630-1750 m. In R. Navardauskas Palaima (Ed.), Lietuvos muziejų rinkiniai (Vol.1, pp. 23-27). Kèdainiai: Kèdainių krašto muziejus. 